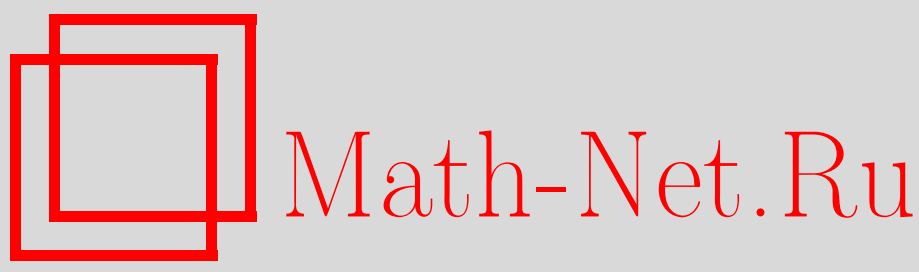

С. А. Шкарин, Бесконечномерное сепарабельное предгильбертово пространство, не гомеоморфное своей замкнутой гиперплоскости, Изв. РАН. Сер. матем., 1999, том 63, выпуск 6, 209-219

DOI: https://doi.org/10.4213/im271

Использование Общероссийского математического портала Math-Net.Ru подразумевает, что вы прочитали и согласны с пользовательским соглашением

http://www. mathnet.ru/rus/agreement

Параметры загрузки:

IP : 35.173 .219 .149

26 апреля 2023 г., 16:57:31 
УДК 517.5

\author{
С. А. Шкарин
}

\title{
Бесконечномерное сепарабельное предгильбертово пространство, не гомеоморфное своей замкнутой гиперплоскости
}

\author{
Построен пример бесконечномерного сепарабельного предгильбертова про- \\ странства, не гомеоморфного своей замкнутой гиперплоскости. \\ Библиография: 11 наименований.
}

\section{Введение}

Согласно теореме Кадеца [1] все сепарабельные бесконечномерные банаховы пространства гомеоморфны. В частности, всякое бесконечномерное сепарабельное банахово пространаство $X$ гомеоморфно своей замкнутой гиперплоскости (или, что то же самое, $X$ гомеоморфно $X \times \mathbb{R}$ ). В работе [2] строится пример сепарабельного бесконечномерного банахова пространства, не изоморфного (как банахово пространство) своей замкнутой гиперплоскости.

Прежде чем сформулировать основной результат работы, напомним несколько определений. Топологическоепространство называется бәровским (см. [3], [4]), если для всякого множества первой категории (счетного объединения нигде не плотных множеств) $A \subset X$ множество $X \backslash A$ плотно в $X$. Для однородных пространств, в частности для топологических групп, это условие эквивалентно тому, что $X$ не является множеством первой категории в себе. Для подмножества $A$ линейного пространства $X$ символом $\operatorname{lin} A$ будем обозначать линейную оболочку множества $A$. Алгебраическая размерность $\operatorname{dim} X$ линейного пространства $X$ - это мощность базиса Гамеля (см. [6], [11]) в $X$. Под топологической размерностью топологического пространства мы будем понимать размерность в смысле $\mathrm{dim}^{1}$.

В настоящей работе строится пример сепарабельного неполного вещественного предгильбертова пространства $E$, обладающего следующими свойствами:

(W1) пространство $E$ является бэровским;

(W2) для всякого непрерьвного отображения $f: E \rightarrow E$ существует счетномерное линейное подпространство $L \subset E$ такое, что $f(x) \in \operatorname{lin}(\{x\} \cup L)$ для всех $x \in E$;

(W3) для всякого непрерывного отображения $f: E \rightarrow E$ найдутся $n \in \mathbb{N}$ и непустое открытое множество $U \subset E$ такие, что $f(x)=a_{0}(x) x+\sum_{j=1}^{n} a_{j}(x) e_{j}$ при всех $x \in U$, где $a_{j}: U \rightarrow \mathbb{R}$ - непрерывные функции и $e_{1}, \ldots, e_{n}-$ линейно независимый набор элементов $E$;

(W4) если $A, B \subset E$ и $A \times B$ является непрерывным образом $E$, то $\min \{\operatorname{dim} \operatorname{lin} A$, $\operatorname{dim} \operatorname{lin} B\} \leqslant \aleph_{0}$

\footnotetext{
${ }^{1}$ Для сепарабельных метризуемых пространств (только этот случай нас и интересует) три известные топологические размерности dim, ind и Ind совпадают (см. [3], [5]).
}

$$
\text { (C) С.А. ШКАРИн } 1999
$$


(W5) для вского неодноточечного топологического пространства $Y$ пространства $E$ и $E \times Y$ не гомеоморфны;

(W6) если $E$ гомеоморфно $Y \times Z$, где $Y$ и $Z$ - топологические пространства, то в одном из этих пространств есть непустое открытое подмножество конечной топологической размерности;

(W7) линейная оболочка всякого подмножества $E$, гомеоморфного $E$, совпадает с $E$;

(W8) всякий линейный непрерывньй оператор $T: E \rightarrow E$ имеет вид $T=\lambda I+F$, где $\lambda \in \mathbb{R}, I$ - тождественный оператор на $E$, а $F$ - конечномерный оператор.

ЗАмЕчАнИЕ. Из условия (W7) следует негомеоморфность $E$ и замкнутой гиперплоскости в $E$. Это (так же, как и (W5)) влечет негомеоморфность $E$ и $E \times \mathbb{R}$. Из (W1) и (W4) вытекает, что $E \times E$ не является непрерывным образом $E$.

В работе [7] построено сепарабельное бесконечномерное предгильбертово пространство, удовлетворяющее условиям (W6) и (W8). В работе [8] построено сепарабельное бесконечномерное предгильбертово пространство, удовлетворяющее условию (W8). В работе [10] построено сепарабельное бесконечномерное предгильбертово пространство $E$ такое, что $E \times E$ не является непрерывным образом $E$ (ослабленный вариант свойства (W4)). Таким образом, наш пример обладает всеми свойствами примеров из [7], [8] и [10] и дополнительно обладает свойствами (W2), (W3), (W5) и (W7).

\section{$\S$ 1. Вспомогательные результаты}

В этой работе все векторные пространства предполагаются вещественными. Напомним, что польским пространством называется сепарабельное метризуемое полной метрикой топологическое пространство. Множество первой категории в топологическом пространстве $X$ будем назьвать тощим в $X$.

Лемма 1. Пусть $X$ - хаусдорфово топологическое пространство, $A \subseteq X-$ всюду плотное множество, $f$-непрерывное отображсние из $A$ в метризуемое полной метрикой топологическое пространство $Ү$. Обозначим

$$
C(f)=\left\{x \in X: \exists \bar{f}(x)=\lim _{\substack{a \rightarrow x \\ a \in A}} f(a)\right\}
$$

Тогда $A \subseteq C(f), C(f)$ - множество типа $G_{\delta}$ в $X,\left.\bar{f}\right|_{A}=f$ и отображение $\bar{f}: C(f) \rightarrow Y$ непрерьвно.

ДокАЗАТЕльство. Пусть $d$ - полная метрика, задающая топологию $Y$. Для всякого $x \in X$ положим $\omega_{f}(x)=\varlimsup_{\lim _{y, z \rightarrow x}} d(f(y), f(z))$. Нетрудно убедиться В тОМ, что

$$
A \subseteq C(f)=\left\{x \in X: \omega_{f}(x)=0\right\}=X \backslash \bigcup_{n=1}^{\infty} B_{n}
$$

где $B_{n}=\left\{x \in X: \omega_{f}(x) \geqslant 1 / n\right\}$, и что множества $B_{n}$ замкнуты. Следовательно, $C(f)$ имеет тип $G_{\delta}$. Соотношение $\left.\bar{f}\right|_{A}=f$ и непрерьвность $\bar{f}$ проверяются элементарно.

Для линейного пространства $Y$ символом $\operatorname{dim} Y$ будем обозначать алгебраическую размерность $Y$. Напомним, что диаметром множества $A \subset X$ в метрическом 
пространстве $(X, d)$ называется число $\operatorname{diam} A=\sup \{d(x, y): x, y \in A\}$. В предположении справедливости континуум-гипотезы следующие две леммы очевидны. Тем не менее они справедливы и без этого предположения.

ЛЕмма 2. Пусть $X$ - польское пространство, $B$ - хаусдорфово топологическое векторное пространство, $f: X \rightarrow B$ - непрерывное отображение. Тогда либо $\operatorname{dim} \operatorname{lin}(f(X)) \leqslant \aleph_{0}$, либо $\operatorname{dim} \operatorname{lin}(f(X))=\mathfrak{c}\left(\right.$ здесь и ниже $\mathfrak{c}=2^{\aleph_{0}}$ мощность континуума).

ДокаЗАТЕЛЬСТво. Пусть $G$ - множество тех $x \in X$, для которых существует окрестность $U_{x}$ такая, что $\operatorname{dim} \operatorname{lin}\left(f\left(U_{x}\right)\right) \leqslant \aleph_{0}$. Ясно, что $G$ открыто и что $\left\{U_{x}: x \in G\right\}$ - открытое покрытие $G$. Так как $G$ метризуемо и сепарабельно, из этого покрытия можно выделить счетное подпокрытие $\left\{U_{x_{n}}: n \in \mathbb{N}\right\}$. Тогда

$$
\operatorname{dim} \operatorname{lin} f(G)=\operatorname{dim} \operatorname{lin}\left(\bigcup_{n=1}^{\infty} f\left(U_{x_{n}}\right)\right) \leqslant \aleph_{0} .
$$

Таким образом, если $G=X$, то доказывать нечего. Пусть $F=X \backslash G \neq \varnothing$. Если $x$ - изолированная точка множества $F$, то существует окрестность $U$ точки $x$ в $X$ такая, что $U \backslash\{x\} \subset G$. Тогда $\operatorname{lin}(f(U)) \subseteq \operatorname{lin}(f(G) \cup\{f(x)\})$. Значит, $\operatorname{dim} \operatorname{lin}(f(U)) \leqslant \aleph_{0}$, что противоречит включению $x \in F$. Следовательно, множество $F$ совершенно (не имеет изолированных точек). Зафиксируем полную метрику $d$, задающую топологию $X$. Пусть

$$
D=\bigcup_{n=1}^{\infty}\{0,1\}^{n}
$$

Построим индуктивно набор $\left\{U_{\alpha}: \alpha \in D\right\}$ открытых подмножеств $X$, обладающих следующими свойствами:

(S1) $\bar{U}_{\alpha} \subset U_{\alpha_{1}, \ldots, \alpha_{n-1}}$ при всех $n \geqslant 2$ и $\alpha \in\{0,1\}^{n}$;

(S2) $\bar{U}_{\alpha} \cap \bar{U}_{\beta}=\varnothing$ при всех $n \in \mathbb{N}$ и $\alpha, \beta \in\{0,1\}^{n}$ таких, что $\alpha \neq \beta$;

(S3) набор векторов $\left\{f\left(x_{\alpha}\right): \alpha \in\{0,1\}^{n}\right\}$ линейно независим при всех $n \in \mathbb{N}$ и любых $x_{\alpha} \in U_{\alpha}$;

(S4) $\operatorname{diam} U_{\alpha} \leqslant 1 / n$ при всех $n \in \mathbb{N}$ и $\alpha \in\{0,1\}^{n}$;

(S5) $U_{\alpha} \cap F \neq \varnothing$ для всех $n \in \mathbb{N}$ и $\alpha \in\{0,1\}^{n}$.

При построении мы будем много раз неявно пользоваться следующим свойством, вытекающим из хаусдорфовости $B$. А именно, если $v_{1}, \ldots, v_{n}-$ линейно независимый набор элементов $B$, то существуют $V_{1}, \ldots, V_{n}$ - окрестности соответственно точек $v_{1}, \ldots, v_{n}$ такие, что для любых $w_{i} \in V_{i}(1 \leqslant i \leqslant n)$ векторы $w_{1}, \ldots, w_{n}$ тоже линейно независимы.

Основание индукции. Из определения $F$ видно, что $\operatorname{dim} \operatorname{lin}(f(U))>\aleph_{0}$ для всякого $x \in F$ и любой окрестности $U$ точки $x$ в $X$. Так как $\operatorname{dim} \operatorname{lin}(f(G))<\aleph_{0}$, имеем, что $\operatorname{dim} \operatorname{lin}(f(U \cap F))>\aleph_{0}$. В частности, сушествует пара точек $y_{0}, y_{1} \in F$ такая, что векторы $f\left(y_{0}\right)$ и $f\left(y_{1}\right)$ линейно независимы. В силу непрерывности $f$ существуют окрестности $U_{0}$ и $U_{1}$ соотвественно точек $y_{0}$ и $y_{1}$ в $X$ такие, что для любых $x_{0} \in U_{0}$ и $x_{1} \in U_{1}$ векторы $f\left(x_{0}\right)$ и $f\left(x_{1}\right)$ линейно независимы. При этом можно сделать так, чтобы $\operatorname{diam} U_{0} \leqslant 1 / 2, \operatorname{diam} U_{1} \leqslant 1 / 2$ и $\bar{U}_{0} \cap \bar{U}_{1}=\varnothing$.

Шаг индукции. Пусть открытые множества $U_{\alpha}$ для $\alpha \in \bigcup_{k=1}^{n-1}\{0,1\}^{k}$, удовлетворяюшие требуемым условиям, уже построены. Построим $U_{\alpha}$ для $\alpha \in\{0,1\}^{n}$. 
Пусть $\{0,1\}^{n-1}=\left\{\alpha^{1}, \ldots, \alpha^{2^{n-1}}\right\}$. Так как $\operatorname{dim} \operatorname{lin} f\left(U_{\alpha^{1}} \cap F\right)>\aleph_{0}$, существуют $y_{\alpha^{1}, 0}, y_{\alpha^{1}, 1} \in U_{\alpha^{1}} \cap F$ такие, что векторы $f\left(y_{\alpha^{1}, 0}\right)$ и $f\left(y_{\alpha^{1}, 1}\right)$ линейно независимы. Так как $\operatorname{dim} \operatorname{lin} f\left(U_{\alpha^{2}} \cap F\right)>\aleph_{0}$, сушествуют $y_{\alpha^{2}, 0}, y_{\alpha^{2}, 1} \in U_{\alpha^{2}} \cap F$ такие, что набор векторов $\left\{f\left(y_{\alpha^{1}, 0}\right), f\left(y_{\alpha^{1}, 1}\right), f\left(y_{\alpha^{2}, 0}\right), f\left(y_{\alpha^{2}, 1}\right)\right\}$ линейно независим. Действуя далее так же, мы получим множество $\left\{y_{\alpha, i}: \alpha \in\{0,1\}^{n-1}, i \in\{0,1\}\right\}$ такое, что $y_{\alpha, i} \in U_{\alpha}$ для всех $\alpha \in\{0,1\}^{n-1}, i \in\{0,1\}$ и набор векторов $\left\{f\left(y_{\alpha, i}\right): \alpha \in\right.$ $\left.\{0,1\}^{n-1}, i \in\{0,1\}\right\}$ линейно независим. В силу непрерьвности $f$ существуют окрестности $U_{\alpha}$ точек $y_{\alpha}\left(\alpha \in\{0,1\}^{n}\right)$ такие, что для любых $x_{\alpha} \in U_{\alpha}$ набор векторов $\left\{f\left(x_{\alpha}\right): \alpha \in\{0,1\}^{n}\right\}$ линейно независим. При этом (уменьшая, если надо, окрестности $\left.U_{\alpha}\right)$ можно сделать так, чтобы $\bar{U}_{\alpha} \subset U_{\left(\alpha_{1}, \ldots, \alpha_{n-1}\right)}, \operatorname{diam} U_{\alpha} \leqslant 1 / n$ и $\bar{U}_{\alpha} \cap \bar{U}_{\beta}=\varnothing$ при $\alpha, \beta \in\{0,1\}^{n}, \alpha \neq \beta$. Построение набора $U_{\alpha}$ завершено.

В силу условий (S1), $(\mathrm{S} 4)$ и полноты метрического пространства $(X, d)$ для всякого $\alpha \in\{0,1\}^{\mathbb{N}}$ множество $\bigcap_{n=1}^{\infty} U_{\left(\alpha_{1}, \ldots, \alpha_{n}\right)}$ состоит из одной точки $x_{\alpha}$. Поскольку $\left|\{0,1\}^{\mathbb{N}}\right|=\mathfrak{c}$, для доказательства леммы нам достаточно проверить, что набор векторов $\left\{f\left(x_{\alpha}\right): \alpha \in\{0,1\}^{\mathbb{N}}\right\}$ линейно независим (то, что $\operatorname{dim} \operatorname{lin}(f(X)) \leqslant \mathfrak{c}$, следует из сепарабельности $X)$. Для этого достаточно показать, что для любого конечного множества $\left\{\alpha^{1}, \ldots, \alpha^{m}\right\} \subset\{0,1\}^{\mathbb{N}}$ набор векторов $\left\{f\left(x_{\alpha^{j}}\right): 1 \leqslant j \leqslant m\right\}$ линейно независим. Выберем $N \in \mathbb{N}$ так, чтобы $\left(\alpha_{1}^{i}, \ldots, \alpha_{N}^{i}\right) \neq\left(\alpha_{1}^{j}, \ldots, \alpha_{N}^{j}\right)$ при $1 \leqslant i<j \leqslant m$. Линейная независимость $f\left(x_{\alpha^{i}}\right)$ вытекает из (S3), так как $x_{\alpha^{i}} \in U_{\left(\alpha_{1}^{i}, \ldots, \alpha_{N}^{i}\right)}$ при всех $i$.

ЛЕмма 3. Пусть $X$ - польское пространство, $B$ - хаусдорфово топологическое векторное пространство, $f, g: X \rightarrow B$ - непрерывные отображения $u$

$$
\tau=\min \left\{\operatorname{dim} L: \begin{array}{l}
L-\text { линейное подпространство B } u \\
f(x) \in \operatorname{lin}(\{g(x)\} \cup L) \text { при всех } x \in X
\end{array}\right\} .
$$

Тогда либо $\tau \leqslant \aleph_{0}$, либо $\tau=\mathfrak{c}$.

ДокАЗАТЕЛЬСТво. Пусть $G$-множество тех $x \in X$, для которых сушествуют окрестность $U_{x}$ и не более чем счетномерное линейное подпространство $L_{x} \subset B$ такие, что $f(y) \in \operatorname{lin}\left(\{g(y)\} \cap L_{x}\right)$ при всех $y \in U_{x}$. Ясно, что $G$ открыто и что $\left\{U_{x}: x \in G\right\}$ - открытое покрытие $G$. Так как $G$ метризуемо и сепарабельно, из этого покрытия можно выделить счетное подпокрытие $\left\{U_{x_{n}}: n \in \mathbb{N}\right\}$. Положим $L=\operatorname{lin}\left(\bigcup_{n=1}^{\infty} L_{x_{n}}\right)$. Тогда $\operatorname{dim} L \leqslant \aleph_{0}$ и $f(y) \in \operatorname{lin}(\{g(y)\} \cap L)$ для всех $y \in G$. Стало быть, если $G=X$, то $\tau \leqslant \aleph_{0}$. Покажем, что $\tau=\mathfrak{c}$ при $G \neq X$. Пусть $F=$ $X \backslash G \neq \varnothing$. Так же, как в доказательстве предыдущей леммы, можно проверить, что множество $F$ совершенно. Кроме того, для всякого открытого $U \subset X$ такого, что $U \cap F \neq \varnothing$, имеем $\operatorname{dim} \operatorname{lin}(f(U \cap F))>\aleph_{0}$ (иначе положим $L_{1}=\operatorname{lin}(f(U \cap F))$, и тогда $f(x) \in \operatorname{lin}\left(\{g(x)\} \cup\left(L+L_{1}\right)\right)$ при всех $x \in U$ и $\operatorname{dim}\left(L+L_{1}\right) \leqslant \aleph_{0}$, что противоречит непустоте $U \cap F)$. Возможны два случая.

Случай 1. Существует открытое множество $U \subset X$ такое, что $U \cap F \neq \varnothing$ и $\operatorname{dim} \operatorname{lin} g(U \cap F) \leqslant \aleph_{0}$.

Множество $U \cap F$ является польским пространством как $G_{\delta}$-подмножество польского пространства (см. [3]). Значит, по лемме 2 существует континуальное подмножество $A \subset U \cap F$ такое, что отображение $\left.f\right|_{A}$ инъективно и набор векторов $\{f(x): x \in A\}$ линейно независим. Если $L_{2}$ - такое линейное подпространство $B$, что $f(x) \in \operatorname{lin}\left(\{g(x)\} \cup L_{2}\right)$ при всех $x \in X$, то $f(A) \subset \operatorname{lin}(g(U \cap F))+L_{2}$. Поскольку 
множество $f(A)$ линейно независимо и континуально, a $\operatorname{lin}(g(U \cap F))$ счетномерно, имеем $\operatorname{dim} L_{2} \geqslant \mathfrak{c}$. Значит, $\tau=\mathfrak{c}$.

Случай 2. Для всякого открытого $U$ такого, что $U \cap F \neq \varnothing$, выполняется неравенство $\operatorname{dim} \operatorname{lin} g(U \cap F)>\aleph_{0}$.

В этом случае так же, как при доказательстве леммы 2 , можно индуктивно построить набор $\left\{U_{\alpha}: \alpha \in D\right\}$ ( $D$ определяется формулой (1)) открытых подмножеств $X$, обладаюших свойствами (S1), $(\mathrm{S} 2),(\mathrm{S} 4),(\mathrm{S} 5)$ (с новым $F$ ) и

$\left(\mathrm{S} 3^{\prime}\right)$ набор векторов $\left\{f\left(x_{\alpha}\right), g\left(x_{\alpha}\right): \alpha \in\{0,1\}^{n}\right\}$ линейно независим при всех $n \in \mathbb{N}$ и любых $x_{\alpha} \in U_{\alpha}$.

Далее, так же, как в доказательстве леммы 2 , можно показать, что набор векторов $\left\{f\left(x_{\alpha}\right), g\left(x_{\alpha}\right): \alpha \in\{0,1\}^{\mathbb{N}}\right\}$ (здесь $\left.\left\{x_{\alpha}\right\}=\bigcap_{n=1}^{\infty} U_{\left(\alpha_{1}, \ldots, \alpha_{n}\right)}\right)$ линейно независим. В силу континуальности множества $\{0,1\}^{\mathbb{N}}$ для всякого линейного подпространства $L \subset B$ размерности меньше $\mathfrak{c}$ найдется $\alpha \in\{0,1\}^{\mathbb{N}}$ такое, что $\operatorname{dim} \operatorname{lin}\left(L \cup\left\{f\left(x_{\alpha}\right), g\left(x_{\alpha}\right)\right\}\right) / L=2$. Тем самым $f\left(x_{\alpha}\right) \notin \operatorname{lin}\left(L \cup\left\{g\left(x_{\alpha}\right)\right\}\right)$. Следовательно, $\tau=\mathfrak{c}$.

\section{§ 2. Основная конструкция}

Перейдем к построению нашего пространства. При этом мы зафиксируем некоторые обозначения, которыми будем пользоваться в дальнейшем без дополнительных разъяснений. Пусть $X$ - полное сепарабельное бесконечномерное метризуемое топологическое векторное пространство, $A \subset X$ - счетное всюду плотное множество, $\mathscr{F}$ - множество всех непрерывных отображений из $A$ в $X$. Очевидно, $\mathscr{F}$ - множество мошности с. Значит, $\mathscr{F}$ можно занумеровать ординалами, меньшими первого континуального: $\mathscr{F}=\left\{f_{\alpha}: 1 \leqslant \alpha<\mathfrak{c}\right\}$. Пусть $\mathscr{K}=\left\{K \subset X: K\right.$ типа $G_{\delta}$ в $X$ и $\left.\operatorname{dim} \operatorname{lin} K=\mathfrak{c}\right\}$. Легко видеть, что $|\mathscr{K}|=\mathfrak{c}$, значит, $\mathscr{K}=\left\{K_{\alpha}: 1 \leqslant \alpha<\mathfrak{c}\right\}$. Опишем индуктивный процесс построения линейных подпространств $E_{\alpha}$ и $G_{\alpha}(\alpha<\mathfrak{c})$ пространства $X$ таких, что $E_{0}=\operatorname{lin} A, G_{0}=\{0\}$ и для всякого $\alpha<\mathfrak{c}$ выполняются следующие соотношения:

(I1) если $\beta<\alpha$, то $E_{\beta} \subseteq E_{\alpha}$ и $G_{\beta} \subseteq G_{\alpha}$;

(I2) $\max \left\{\operatorname{dim} E_{\alpha}, \operatorname{dim} G_{\alpha}\right\} \leqslant \max \left\{\aleph_{0},|\alpha|\right\}$ и $E_{\alpha}$ плотно в $X$;

(I3) $E_{\alpha} \cap G_{\alpha}=\{0\}$ и $E_{\alpha} \cap K_{\alpha} \neq \varnothing$;

(I4) выполняется хотя бы одно из следующих трех условий:

(U1) $E_{\alpha} \backslash C\left(f_{\alpha}\right) \neq \varnothing$ (множество $C\left(f_{\alpha}\right)$ определено формулой $\left.(\alpha)\right)$;

(U2) существует $x_{\alpha} \in E_{\alpha} \cap C\left(f_{\alpha}\right)$ такое, что $\bar{f}_{\alpha}\left(x_{\alpha}\right) \in\left(G_{\alpha}+E_{\alpha}\right) \backslash E_{\alpha}$ (отображение $\bar{f}_{\alpha}$ определено формулой $(\alpha)$ );

(U3) $E_{\alpha} \subset C\left(f_{\alpha}\right)$, и существует счетномерное линейное подпространство $L_{\alpha} \subset X$ такое, что $\bar{f}_{\alpha}(x) \in \operatorname{lin}\left(L_{\alpha} \cup\{x\}\right)$ для всякого $x \in C\left(f_{\alpha}\right)$.

Пусть $1 \leqslant \alpha<\mathfrak{c}$ и линейные подпространства $E_{\beta}, G_{\beta} \subset X$ при $\beta<\alpha$, удовлетворяюшие условиям (I1)-(I4), уже построены. Построим $E_{\alpha}$ и $G_{\alpha}$. Положим

$$
E_{\alpha}^{\prime}=\bigcup_{\beta<\alpha} E_{\beta}, \quad G_{\alpha}^{\prime}=\bigcup_{\beta<\alpha} G_{\beta}
$$

Ясно, что $E_{\alpha}^{\prime}$ и $G_{\alpha}^{\prime}$ - линейные подпространства $X, E_{\alpha}^{\prime} \cap G_{\alpha}^{\prime}=\{0\}$ и $\max \left\{\operatorname{dim} E_{\alpha}^{\prime}, \operatorname{dim} G_{\alpha}^{\prime}\right\} \leqslant \max \left\{\aleph_{0},|\alpha|\right\}$. Возможны 2 случая.

Случай 1 . Существует $x_{\alpha} \in C\left(f_{\alpha}\right)$ такой, что $y_{\alpha}=\bar{f}_{\alpha}\left(x_{\alpha}\right) \notin \operatorname{lin}\left(E_{\alpha}^{\prime} \cup\left\{x_{\alpha}\right\}\right)$. 
Так как $\operatorname{dim} \operatorname{lin} K_{\alpha}=\mathfrak{c}$ и $\max \left\{\operatorname{dim} E_{\alpha}^{\prime}, \operatorname{dim} G_{\alpha}^{\prime}\right\} \leqslant \max \left\{\aleph_{0},|\alpha|\right\}$, найдется $z_{\alpha} \in K_{\alpha} \backslash \operatorname{lin}\left(E_{\alpha}^{\prime} \cup G_{\alpha}^{\prime} \cup\left\{x_{\alpha}, y_{\alpha}\right\}\right)$. Положим $E_{\alpha}=\operatorname{lin}\left(E_{\alpha}^{\prime} \cup\left\{x_{\alpha}, z_{\alpha}\right\}\right)$. Если $y_{\alpha} \in E_{\alpha}+G_{\alpha}^{\prime}$, то положим $G_{\alpha}=G_{\alpha}^{\prime}$. Если $y_{\alpha} \notin E_{\alpha}+G_{\alpha}^{\prime}$, то положим $G_{\alpha}=\operatorname{lin}\left(G_{\alpha}^{\prime} \cup\left\{y_{\alpha}\right\}\right)$. Справедливость соотношений (I1)-(I3) и (U2) для $E_{\alpha}$ и $G_{\alpha}$ проверяется элементарно.

Случай 2. $\bar{f}_{\alpha}(x) \in \operatorname{lin}\left(E_{\alpha}^{\prime} \cup\{x\}\right)$ для всякого $x \in C\left(f_{\alpha}\right)$.

Так как $\operatorname{dim} \operatorname{lin} K_{\alpha}=\mathfrak{c}$ и $\max \left\{\operatorname{dim} E_{\alpha}^{\prime}, \operatorname{dim} G_{\alpha}^{\prime}\right\} \leqslant \max \left\{\aleph_{0},|\alpha|\right\}$, найдется $z_{\alpha} \in K_{\alpha} \backslash\left(E_{\alpha}^{\prime}+G_{\alpha}^{\prime}\right)$. Положим $E_{\alpha}=\operatorname{lin}\left(E_{\alpha}^{\prime} \cup\left\{z_{\alpha}\right\}\right), G_{\alpha}=G_{\alpha}^{\prime}$. Справедливость соотношений (I1)-(I3) для $E_{\alpha}$ и $G_{\alpha}$ очевидна.

Если $E_{\alpha} \backslash C\left(f_{\alpha}\right) \neq \varnothing$, то вьполняется условие (U1). Пусть $E_{\alpha} \subset C\left(f_{\alpha}\right)$. Нам осталось показать выполнение условия (U3) в этом случае.

Согласно лемме $1 C\left(f_{\alpha}\right)$ - множество типа $G_{\delta}$ в $X$, а значит (см. [3]), $C\left(f_{\alpha}\right)$ является польским пространством (в индуцированной из $X$ топологии). Пусть $f, g: C\left(f_{\alpha}\right) \rightarrow X$ определяются формулами $f(x)=\bar{f}_{\alpha}(x)$ и $g(x)=x$. Тогда $f$ и $g$ непрерывны ( $f$ непрерывна по лемме 1$)$ и, значит, по лемме 3 , примененной к паре функций $f$ и $g$, кардинал

$$
\tau=\min \left\{\operatorname{dim} L: \quad \begin{array}{l}
L-\text { линейное подпространство } X \text { и } \\
\bar{f}_{\alpha}(x) \in \operatorname{lin}(\{x\} \cup L) \text { при всех } x \in C\left(f_{\alpha}\right)
\end{array}\right\}
$$

либо не более чем счетен, либо равен с. Но так как $\bar{f}_{\alpha}(x) \in \operatorname{lin}\left(E_{\alpha}^{\prime} \cup\{x\}\right)$ для всякого $x \in C\left(f_{\alpha}\right)$, имеем, что $\tau \leqslant \operatorname{dim} E_{\alpha}^{\prime} \leqslant \max \left\{\aleph_{0},|\alpha|\right\}<\mathfrak{c}$. Значит, $\tau \leqslant \aleph_{0}$. Таким образом, сушествует счетномерное линейное подпространство $L_{\alpha} \subset X$ такое, что $\bar{f}_{\alpha}(x) \in \operatorname{lin}\left(L_{\alpha} \cup\{x\}\right)$ при всех $x \in C\left(f_{\alpha}\right)$. Условие (U3) проверено.

Построение $E_{\alpha}$ и $G_{\alpha}$ завершено. Пусть теперь $E_{X}=\bigcup_{\alpha<\mathfrak{c}} E_{\alpha}$. Тогда $E_{X}-$ плотное линейное подпространство $X$. Ясно, что $E_{X}$ с топологией, индуцированной из $X$, является бесконечномерным сепарабельным метризуемым топологическим векторным пространством. Заметим, что если $X=l_{2}$, то $E_{X}$ - предгильбертово пространство.

\section{$\S$ 3. Свойства пространства $E_{X}$}

\section{ПРЕДЛОЖЕНИЕ 1. Пространство $E_{X}$ является бәровским.}

ДокАЗАТЕЛЬСТво. Предположим, что $E_{X}$ не является бэровским. Так как всякое однородное небэровское пространство является множеством первой категории в себе, сушествуют замкнутые нигде не плотные множества $F_{n} \subset X$ такие, что $E_{X} \subset \bigcup_{n=1}^{\infty} F_{n}$. Положим $G=X \backslash \bigcup_{n=1}^{\infty} F_{n}$. Тогда $G$ - множество типа $G_{\delta}$ в $X$ и, значит, $G$ является польским пространством. Пусть $f: G \rightarrow X, f(x)=x-$ тождественное вложение. Очевидно, $f$ непрерьвно. По лемме 2 либо $\operatorname{dim} \operatorname{lin} G=\mathfrak{c}$, либо $\operatorname{dim} \operatorname{lin} G \leqslant \aleph_{0}$. Если $\operatorname{dim} \operatorname{lin} G \leqslant \aleph_{0}$, то $\operatorname{lin} G$ является объединением последовательности конечномерных линейных подпространств $X$. Следовательно, $\operatorname{lin} G$ и тем более само $G$ являются тошими в $X$. Но тогда $X=G \cup \bigcup_{n=1}^{\infty} F_{n}$ является тощим в себе, что противоречит теореме Бэра. Если же $\operatorname{dim} \operatorname{lin} G=\mathfrak{c}$, то $G \in \mathscr{K}$. Значит, найдется ординал $1 \leqslant \alpha<\mathfrak{c}$ такой, что $G=K_{\alpha}$. В силу (I3) $G \cap E_{\alpha} \neq \varnothing$, что противоречит включению $E_{\alpha} \subset E_{X} \subset \bigcup_{n=1}^{\infty} F_{n}$. 
ПРЕДЛОЖЕНИЕ 2. Для всякого непрерывного отображсения $f: E_{X} \rightarrow E_{X}$ найдется счетномерное линейное подпространство $L \subset E_{X}$ такое, что $f(x) \in \operatorname{lin}(\{x\} \cup L)$ при всех $x \in E_{X}$. Кроме того, для всякого непустого открытого множества $V \subseteq X$ найдутся $n \in \mathbb{N}$ u непустое открытое множество $U \subseteq X$ такие, что $U \subseteq V$ и

$$
\bar{f}(x)=a_{0}(x) x+\sum_{j=1}^{n} a_{j}(x) e_{j}
$$

при всех $x \in U \cap C(f)$, где $a_{j}: U \cap C(f) \rightarrow \mathbb{R}$ - непрерывные функиии, $e_{1}, \ldots, e_{n}-$ линейно независимый набор әлементов $E_{X}(\bar{f}$ u $C(f)$ определяются формулой $(\alpha))$.

ДоказАТЕльСтво. Так как $\left.f\right|_{A}$ является непрерывньм отображением из $A$ в $X$, найдется ординал $\alpha$ такой, что $1 \leqslant \alpha<\mathfrak{c}$ и $\left.f\right|_{A}=f_{\alpha}$. По построению $E_{X}$ для этого $\alpha$ выполняется по крайней мере одно из условий (U1), (U2) или (U3). В силу непрерывности $f$ на $E_{X}$ имеем, что $E_{\alpha} \subset E_{X} \subset C\left(f_{\alpha}\right)$ и $\left.\bar{f}_{\alpha}\right|_{E_{X}}=f$. Следовательно, условие (U1) не вьполняется. Так как $\bar{f}_{\alpha}\left(E_{\alpha}\right)=f\left(E_{\alpha}\right) \subseteq f\left(E_{X}\right) \subseteq E_{X}$ и $E_{X} \cap\left(E_{\alpha}+G_{\alpha}\right)=E_{\alpha}$, имеем, что условие (U2) для $\alpha$ тоже не выполняется. Стало быть, выполняется (U3). Значит, существует счетномерное линейное подпространство $L_{\alpha} \subset X$ такое, что $\bar{f}(x) \in \operatorname{lin}\left(\{x\} \cup L_{\alpha}\right)$ для всех $x \in C(f)$. Положим $L=L_{\alpha} \cap E_{X}$. Тогда $f(x)=\bar{f}(x) \in E_{X} \cap \operatorname{lin}\left(\{x\} \cup L_{\alpha}\right)=\operatorname{lin}(\{x\} \cup L)$ для всякого $x \in E_{X}$ и $L$ является счетномерным подпространством $E_{X}$. Первое утверждение предложения проверено.

В силу своей счетномерности $L$ является объединением возрастающей последовательности $M_{n}(n \in \mathbb{N})$ конечномерных линейных подпространств $E_{X}$. Пусть $F_{n}=\left\{x \in C(f): \bar{f}(x) \in \operatorname{lin}\left(\{x\} \cup M_{n}\right)\right\}$. Тогда $E_{X} \subseteq \bigcup_{n=1}^{\infty} F_{n}$. По предложению 1 замыкание хотя бы одного из множеств $F_{n} \cap V \cap E_{X}$ имеет непустую внутренность в $V \cap E_{X}$. В силу плотности $E_{X}$ в $X$ и нигде не плотности любого конечномерного линейного подпространства $X$ существуют $m \in \mathbb{N}$ и непустое открытое множество $U \subset X$ такие, что $U \subset V \backslash M_{m}$ и $F_{m} \cap U$ плотно в $U$. Из определения $F_{m}$ видно, что $F_{m} \backslash M_{m}$ замкнуто в $C(f) \backslash M_{m}$. Следовательно, $U \cap C(f) \subseteq F_{m}$. Значит, $\bar{f}(x) \in \operatorname{lin}\left(\{x\} \cup M_{m}\right)$ для всех $x \in U \cap C(f)$. Пусть $\left\{e_{1}, \ldots, e_{n}\right\}-$ базис в $M_{m}$ и $\left\{\alpha_{1}, \ldots, \alpha_{n}\right\}$ - координатные функционалы ${ }^{2}$ этого базиса. Для $x \in U$ символом $\varphi_{x}$ обозначим линейный функционал на пространстве $\operatorname{lin}\left(\{x\} \cup M_{m}\right)$ с ядром $M_{m}$ такой, что $\varphi_{x}(x)=1$. Пусть $x \in U \cap C(f)$. Поскольку $\bar{f}(x) \in \operatorname{lin}(\{x\} \cup M)$, имеем

$$
\bar{f}(x)=\varphi_{x}(\bar{f}(x)) x+\sum_{j=1}^{n} \alpha_{j}\left(\bar{f}(x)-\varphi_{x}(\bar{f}(x)) x\right) e_{j} .
$$

Положим $a_{0}(x)=\varphi_{x}(\bar{f}(x)), a_{j}(x)=\alpha_{j}\left(\bar{f}(x)-\varphi_{x}(\bar{f}(x)) x\right)$ при $1 \leqslant j \leqslant n$. Легко видеть, что функции $a_{j}$ непрерывны на $U \cap C(f)$. Тем самым представление $\bar{f}(x)=a_{0}(x) x+\sum_{j=1}^{n} a_{j}(x) e_{j}$ обладает всеми требуемыми свойствами.

Лемма 4. Пусть множество $A \subseteq \mathbb{R}^{n}$ гомеоморфно $\mathbb{R}^{n}$. Тогда $A$ открыто.

\footnotetext{
кера.

${ }^{2}$ То есть $\alpha_{j}$ - линейные функционалы на $M_{m}$ и $\alpha_{j}\left(e_{i}\right)=\delta_{i, j}$, где $\delta_{i, j}$ - символ Кронек-
} 
ДокАЗАТЕЛЬСТво. Это утверждение непосредственно вытекает из теоремы Урысона-Менгера (см. [6, теорема 1, с. 425]).

При доказательстве следующего предложения нам понадобится понятие множества со свойством Бэра (см. [4], [9]). Говорят, что подмножество $B$ топологического пространства $Y$ обладает свойством Бәра, если $B$ представляется в виде $U \triangle P$, где $U$ - открытое, а $P$ - тощее подмножества $Y$.

ПрЕДЛОЖЕНИЕ 3. Пусть отображение $f: E_{X} \rightarrow E_{X}$ яв.ляется гомеоморфизмом $E_{X}$ и $f\left(E_{X}\right)$. Тогда найдется непустое открытое множество $W \subset E_{X}$ такое, что множество $W \backslash f\left(E_{X}\right)$ является тощим в $E_{X}$.

ДокАЗАТЕльСтво. Согласно предложению 2 существует непустое открытое множество $U \subset X$ такое, что для всех $x \in U \cap C(f)$

$$
\bar{f}(x)=a_{0}(x) x+\sum_{j=1}^{n} a_{j}(x) e_{j},
$$

где $e_{1}, \ldots, e_{n}-$ линейно независимый набор векторов из $E_{X}$, функции $a_{j}: U \cap$ $C(f) \rightarrow \mathbb{R}$ непрерывны. Тогда $a_{0} \not \equiv 0$ (иначе в силу (2) $f: U \cap E_{X} \rightarrow f\left(U \cap E_{X}\right)$ будет гомеоморфизмом пространства $U \cap E_{X}$ бесконечной топологической размерности и пространства $f\left(U \cap E_{X}\right) \subset \operatorname{lin}\left\{e_{1}, \ldots, e_{n}\right\}$ топологической размерности, не превосходящей $n$ ). В силу непрерывности $a_{0}$ можно выбрать непустое открытое множество $V \subset X$ такое, что $V \subset U, V \cap \operatorname{lin}\left\{e_{1}, \ldots, e_{n}\right\}=\varnothing$ и $a_{0}$ на $V \cap C(f)$ не обрашается в нуль. Множество $V \cap C(f)$ является польским пространством как $G_{\delta}$-подмножество польского пространства $X$. Значит, множество $\bar{f}(V \cap C(f)) \subset X$ является аналитическим (см. [9]) как непрерывный образ польского пространства. Следовательно (см. [9]), множество $\bar{f}(V \cap C(f)) \subset X$ обладает свойством Бэра. Значит, множество $E_{X} \cap \bar{f}(V \cap C(f))$ является множеством со свойством Бэра в $E_{X}$. Если $x \in(C(f) \cap V) \backslash E_{X}$, то $a_{0}(x) x \notin E_{X}$, так как $a_{0}(x) \neq 0$, а $\sum_{j=1}^{n} a_{j}(x) e_{j} \in E_{X}$. Следовательно, по $(2) \bar{f}(x) \notin E_{X}$. Значит, $f\left(V \cap E_{X}\right)=E_{X} \cap \bar{f}(V \cap C(f))$. Тем самым, множество $f\left(V \cap E_{X}\right) \subset E_{X}$ обладает свойством Бэра в $E_{X}$. Для всякого множества со свойством Бэра (это непосредственно следует из определения) выполняется альтернатива: либо это множество тощее, либо его дополнение в некотором непустом открытом множестве является тощим. Предположим, что утверждение предложения неверно. Тогда множество $f\left(V \cap E_{X}\right)$ является тошим в $E_{X}$. Пусть $x_{0} \in V \cap E_{X}, x \in E_{X}$. Рассмотрим $L_{x}=\operatorname{lin}\left\{e_{1}, \ldots, e_{n}, x, x_{0}\right\}$. По условию $\left.f\right|_{L_{x} \cap V}$ осушествляет гомеоморфизм $L_{x} \cap V$ и $f\left(L_{x} \cap V\right)$. Так как $L_{x} \cap V$ является открытым непустым $\left(x_{0} \in L_{x} \cap V\right)$ выпуклым подмножеством конечномерного хаусдорфова топологического векторного пространства $L_{x}$, то $L_{x} \cap V$ гомеоморфно $L_{x}=\mathbb{R}^{\operatorname{dim} L_{x}}$ (здесь мы пользуемся тем, что всякое хаусдорфово $n$-мерное топологическое векторное пространство изоморфно $\mathbb{R}^{n}$; см. [6]). По (2) $f\left(L_{x} \cap V\right) \subset L_{x}$. Следовательно, $f\left(L_{x} \cap V\right)$ - подмножество $L_{x}$, гомеоморфное $L_{x}$. По лемме $4 f\left(L_{x} \cap V\right)$ открыто в $L_{x}$. Значит, $f\left(L_{x} \cap V\right)-f\left(x_{0}\right)$ является окрестностью нуля в $L_{x}$. Следовательно, сушествует $c_{x}>0$ такое, что $t x \in f\left(L_{x} \cap V\right)-f\left(x_{0}\right)$ при всех $t \in \mathbb{R},|t|<c_{x}$. В силу произвольности $x \in E_{X}$ имеем

$$
\bigcup_{n=1}^{\infty} n\left(f\left(V \cap E_{X}\right)-f\left(x_{0}\right)\right)=E_{X} .
$$


Но множество $f\left(V \cap E_{X}\right)$ является тошим в $E_{X}$, а сдвиги и растяжения являются автогомеоморфизмами пространства $E_{X}$. Значит, все множества $n\left(f\left(V \cap E_{X}\right)-\right.$ $\left.f\left(x_{0}\right)\right)$ являются тошими в $E_{X}$. Стало быть, пространство $E_{X}$ является тощим в себе как счетное объединение тощих множеств (см. (3)), что противоречит предложению 1.

ТЕОРема. Пространство $E_{X}$ удовлетворяет условиям (W1)-(W8) из введения.

ДокАЗАТЕЛЬСТво.Условия (W1)-(W3) непосредственно вытекают из предложений 1 и 2.

Докажем (W4). Пусть $A, B \subseteq E_{X}$ и $\varphi=(f, g): E_{X} \rightarrow A \times B$ - непрерывная сюръекция. По предложению 2 существует счетномерное линейное подпространство $L \subset E_{X}$ такое, что $\{f(x), g(x)\} \subset \operatorname{lin}(\{x\} \cup L)$ при всех $x \in E_{X}$. Если $\operatorname{dim} \operatorname{lin} A>\aleph_{0}$ и $\operatorname{dim} \operatorname{lin} B>\aleph_{0}$, то найдутся $y \in A$ и $z \in B$ такие, что $\operatorname{dim} \operatorname{lin}(L \cup\{y, z\}) / L=2$. В силу сюръективности $\varphi$ найдется $x \in E_{X}$ такой, что $f(x)=y$ и $g(x)=z$. Стало быть, $\{y, z\} \subset \operatorname{lin}(\{x\} \cup L)$. Значит, $\operatorname{dim} \operatorname{lin}(L \cup\{y, z\}) / L \leqslant 1$. Это противоречие показывает, что либо $\operatorname{dim} \operatorname{lin} A \leqslant \aleph_{0}$, либо $\operatorname{dim} \operatorname{lin} B \leqslant \aleph_{0}$. Справедливость (W4) для $E_{X}$ доказана.

Докажем (W5). Пусть $Y$ - неодноточечное топологическое пространство. Предположим, что существует гомеоморфизм $\varphi: E_{X} \rightarrow E_{X} \times Y$. Из метризуемости и линейной связности $E_{X}$ вытекает метризуемость и линейная связность $E_{X} \times Y$. Значит, $Y$ метризуемо и линейно связно. Следовательно, всякое одноточечное подмножество $Y$ нигде не плотно в $Y$. Пусть $y \in Y$. Рассмотрим множество $B=\varphi^{-1}\left(E_{X} \times\{y\}\right) \subset E_{X}$. Так как $E_{X}$ и $E_{X} \times\{y\}$ гомеоморфны, то $B$ гомеоморфно $E_{X}$. Кроме того, $E_{X} \times\{y\}$ нигде не плотно в $E_{X} \times Y$, так как $\{y\}$ нигде не плотно в $Y$. Значит, $B$ нигде не плотно в $E_{X}$ (гомеоморфизм переводит нигде не плотные множества в нигде не плотные). Существование такого $B$ противоречит предложению 3. Справедливость (W5) доказана.

Докажем (W6). Пусть $Y$ и $Z$-топологические пространства и $\varphi=(f, g): E_{X} \rightarrow$ $Y \times Z$ - гомеоморфизм. Так как $\varphi^{-1}\left(Y \times\left\{z_{0}\right\}\right)$ гомеоморфно $Y$ для всякого $z_{0} \in Z$ и $\varphi^{-1}\left(\left\{y_{0}\right\} \times Z\right)$ гомеоморфно $Z$ для всякого $y_{0} \in Z$, можно считать, что $Y$ и $Z$ подмножества $E_{X}$ (с индуцированной топологией). По уже доказанному для $E_{X}$ свойству (W4) имеем, что либо $\operatorname{dim} \operatorname{lin} Y \leqslant \aleph_{0}$, либо $\operatorname{dim} \operatorname{lin} Z \leqslant \aleph_{0}$. Пусть для определенности $\operatorname{dim} \operatorname{lin} Y \leqslant \aleph_{0}$. По предложению 2 найдется непустое открытое множество $U \subset E_{X}$ такое, что

$$
f(x)=a_{0}(x) x+\sum_{j=1}^{n} a_{j}(x) e_{j}
$$

при всех $x \in U$, где $a_{j}: U \rightarrow \mathbb{R}$ - непрерывные функции, а $e_{j}$ - элементы $E_{X}$. Если $a_{0} \not \equiv 0$, то сушествует непустое открытое множество $V \subset U$, на котором $a_{0}$ не обращается в нуль. Так как $f\left(E_{X}\right)=Y$, имеем, что в силу (4) $x \in \operatorname{lin}\left(Y \cup\left\{e_{1}, \ldots, e_{n}\right\}\right)$ для всякого $x \in V$. Следовательно, $E_{X}=\operatorname{lin} V \subseteq \operatorname{lin}\left(Y \cup\left\{e_{1}, \ldots, e_{n}\right\}\right)$. Значит, $\operatorname{dim} E_{X} \leqslant \aleph_{0}$, что противоречит предложению 1 . Стало быть, $a_{0} \equiv 0$. В силу (4) имеем, что $\operatorname{dim} \operatorname{lin} f(U) \leqslant n$. Стало быть, $f(U)$ является непустым открытым конечномерным подмножеством $Y$. Условие (W6) проверено.

Докажем (W7). Пусть $B \subset E_{X}$ и $B$ гомеоморфно $E_{X}$. По предложению 3 сушествуют уравновешенная открытая окрестность нуля $U$ в $E_{X}$ и $x_{0} \in E_{X}$ 
такие, что множество $K=\left(x_{0}+U\right) \backslash B$ является тощим в $E_{X}$. Покажем, что $U \subseteq B-B$. Пусть $y \in U$. Предположим, что $y \notin B-B$. Тогда $(y+B) \cap B=\varnothing$. Следовательно,

$$
\left(\left(y+x_{0}+U\right) \backslash(y+K)\right) \cap\left(\left(x_{0}+U\right) \backslash K\right)=\varnothing .
$$

В силу уравновешенности $U$ и включения $y \in U$ имеем, что множество $W=(y+$ $\left.x_{0}+U\right) \cap\left(x_{0}+U\right)$ открыто и непусто. По $(5) W \subset(K \cup(y+K))$. Значит, непустое открытое множество $W$ является тощим в $E_{X}$, что противоречит бэровости $E_{X}$. Итак, $B-B$ содержит окрестность нуля. Следовательно, линейная оболочка $B$ совпадает с $E_{X}$. Справедливость (W7) доказана.

Докажем (W8). Пусть $T: E_{X} \rightarrow E_{X}$ - непрерывный линейный оператор. По предложению 2 найдутся уравновешенная окрестность нуля $U$ в $E_{X}, e_{0} \in E_{X}$ и линейно независимый набор векторов $\left\{e_{1}, \ldots, e_{n}\right\} \subset E_{X}$ такие, что $\left(e_{0}+U\right) \cap$ $\operatorname{lin}\left\{e_{1}, \ldots, e_{n}\right\}=\varnothing$ и

$$
T x=a(x) x+\sum_{j=1}^{n} a_{j}(x) e_{j}
$$

при всех $x \in e_{0}+U$, где $a: e_{0}+U \rightarrow \mathbb{R}, a_{j}: e_{0}+U \rightarrow \mathbb{R}$ - непрерьвные функции. Тогда для $x \in U$ по (6) имеем

$$
\begin{aligned}
T\left(x+e_{0}\right) & =a\left(x+e_{0}\right)\left(x+e_{0}\right)+\sum_{j=1}^{n} a_{j}\left(x+e_{0}\right) e_{j}, \\
T e_{0} & =a\left(e_{0}\right) e_{0}+\sum_{j=1}^{n} a_{j}\left(e_{0}\right) e_{j} .
\end{aligned}
$$

Вычитая друг из друга последние два равенства, имеем, что для всякого $x \in U$

$$
T x=b(x) x+\sum_{j=0}^{n} b_{j}(x) e_{j},
$$

где $b(x)=a\left(x+e_{0}\right), b_{0}(x)=a\left(x+e_{0}\right)-a\left(e_{0}\right), b_{j}(x)=a_{j}\left(x+e_{0}\right)-a_{j}\left(e_{0}\right)$ при $1 \leqslant j \leqslant n$ - непрерывные функции на $U$. Пусть $L=\operatorname{lin}\left\{e_{0}, \ldots, e_{n}\right\}, x \in U \backslash L$ и $t \in \mathbb{R} \backslash\{0\}$ таково, что $t x \in U$. В силу линейности оператора $T$ и формулы (7) имеем

$$
t b(t x) x+\sum_{j=0}^{n} b_{j}(t x) e_{j}=t b(x) x+\sum_{j=0}^{n} t b_{j}(x) e_{j} .
$$

Поскольку векторы $x, e_{0}, \ldots, e_{n}$ линейно независимы, $b(t x)=b(x)$. Переходя к пределу при $t \rightarrow 0$, в силу непрерывности $b$ имеем, что $b(x)=b(0)$ при всех $x \in U \backslash L$. Из плотности $U \backslash L$ в $U$ и непрерывности $b$ следует, что $b(x)=b(0)$ при всех $x \in U$. Рассмотрим линейный непрерьвньй оператор $F: E_{X} \rightarrow E_{X}$, $F x=T x-b(0) x$. Тогда из (7) и только что полученного соотношения вытекает, что $F x=\sum_{j=0}^{n} b_{j}(x) e_{j}$ для всех $x \in U$. Стало быть, $F(U) \subset L$. Так как $U-$ окрестность нуля в $E_{X}$, а $F$ - линейньй оператор, то $F\left(E_{X}\right) \subset L$. Значит, оператор $F$ конечномерен. Поскольку $(T-F) x=b(0) x$ при $x \in U$ и оператор $T-F$ линеен, то $T-F=b(0) I$. Итак, $T=b(0) I+F$. Справедливость (W8) доказана. 
СлеДСТВИЕ 1. Пространство $E_{l_{2}}$ является сепарабельным предгильбертовым пространством, удовлетворяющим условиям (W1)-(W8).

СлЕДСТВИЕ 2. Если $X=L_{0}[0,1]$ - пространство всех измеримых по Лебегу функиий на $[0,1]$ (точнее, пространство классов эквивалентности относительно равенства почти всюду) с топологией сходимости по мере, то $E=E_{X}$ - сепарабельное метризуемое бесконечномерное топологическое векторное пространство, обладающее следующим забавным свойством:

$\left(W 8^{\prime}\right)$ всякий непрерывный линейный оператор $T: E \rightarrow E$ является оператором умножения на число.

ДоКАЗАТЕЛЬСТВО. Это вытекает из только что доказанной теоремы (свойство $(\mathrm{W} 8))$ и того факта, что на пространстве $L_{0}[0,1]$ не существует ненулевых непрерывных линейных функционалов (см. [11]).

В заключение отметим три интересные нерешенные задачи, связанные с рассмотренной темой.

1. Существует ли аналитическое (см. [9]) бесконечномерное предгильбертово пространство, не гомеоморфное своей замкнутой гиперплоскости?

2. Существует ли полное сепарабельное метризуемое топологическое векторное пространство размерности больше единицы, удовлетворяюшее условию (W $\left.8^{\prime}\right)$ ?

3. Существует ли сепарабельное предгильбертово пространство $E$ такое, что $E$ гомеоморфно $E \times \mathbb{R}^{2}$, но не гомеоморфно $E \times \mathbb{R}$ ?

Автор благодарен О.Г. Смолянову и А. И. Штерну за внимание к работе.

\section{Список литературы}

1. Кадеи, М. И. Доказательство топологической эквивалентности всех сепарабельных бесконечномерных пространств Банаха // Функц. анализ и его прилож. 1967. Т. 1. № 1. C. $61-70$.

2. Gowers W. T., Maurey B. The unconditional basis sequence problem // J. Amer. Math. Soc. 1993. № 4. P. 851-874.

3. Энгелькинг Р. Общая топология. М.: Мир, 1986.

4. Окстоби Дж. Мера и категория. М.: Мир, 1976.

5. Александров П. С., Пасынков Б. А. Введение в теорию размерности. М.: Наука, 1975.

6. Робертсон A., Робертсон В. Топологические векторные пространства. М.: Мир, 1967.

7. Pol $R$. An infinite dimensional pre-Hilbert space non-homeomorphic to its owm square // Proc. Amer. Math. Soc. 1984. V. 90. № 3. P. 450-454.

8. Van-Mill J. An infinite dimensional pre-Hilbert space all bounded linear operators on which are simple // Colloq. Math. 1987. V. 54. № 1. P. 29-37.

9. Куратовский К. Общая топология. Т. 1. М.: Мир, 1966.

10. Marciszewski $W$. An infinite dimensional pre-Hilbert space without a continuous map on its own square // Bull. Acad. Polon. Sci. Ser. math. 1983. V. 31. № 9. P. 393-396.

11. Шефер Х. Топологические векторные пространства. М.: Мир, 1971.

Московский Государственный Университет

им. М. Ю. Ломоносова, механико-математический факультет

E-mail: stas@graal.math.msu.su
Поступило в редакцию

15.IV.1999 